\title{
Modeling and Output Feedback Control of Networked Control Systems with Both Time Delays; and Packet Dropouts
}

\author{
Li Qiu, ${ }^{1,2}$ Qin Luo, ${ }^{1}$ Shanbin $\mathrm{Li}^{2}$, and Bugong $\mathrm{Xu}^{2}$ \\ ${ }^{1}$ Shenzhen Key Laboratory of Urban Rail Transit, College of Mechatronics and Control Engineering, \\ Shenzhen University, Shenzhen 518060, China \\ ${ }^{2}$ Key Laboratory of Autonomous Systems and Network Control, Ministry of Education, \\ School of Control Science and Engineering, South China University of Technology, Guangzhou 510640, China
}

Correspondence should be addressed to Li Qiu; qiuli@szu.edu.cn

Received 12 January 2013; Accepted 13 March 2013

Academic Editor: Yang Tang

Copyright (C) $2013 \mathrm{Li}$ Qiu et al. This is an open access article distributed under the Creative Commons Attribution License, which permits unrestricted use, distribution, and reproduction in any medium, provided the original work is properly cited.

\begin{abstract}
This paper is concerned with the problem of modeling and output feedback controller design for a class of discrete-time networked control systems (NCSs) with time delays and packet dropouts. A Markovian jumping method is proposed to deal with random time delays and packet dropouts. Different from the previous studies on the issue, the characteristics of networked communication delays and packet dropouts can be truly reflected by the unified model; namely, both sensor-to-controller (S-C) and controller-toactuator (C-A) time delays, and packet dropouts are modeled and their history behavior is described by multiple Markov chains. The resulting closed-loop system is described by a new Markovian jump linear system (MJLS) with Markov delays model. Based on Lyapunov stability theory and linear matrix inequality (LMI) method, sufficient conditions of the stochastic stability and output feedback controller design method for NCSs with random time delays and packet dropouts are presented. A numerical example is given to illustrate the effectiveness of the proposed method.
\end{abstract}

\section{Introduction}

Networked control systems (NCSs) are systems in which control loops are closed over a real-time communication network. The fact that controllers, sensors, and actuators are not connected through point-to-point connections but through a multipurpose network offers advantages, such as low cost, decreased wiring, ease of installation and maintenance, and high efficiency, reliability, and flexibility [15]. Consequently, NCSs are applied in a broad range such as manufacturing plants, vehicles, aircrafts, spacecrafts, and remote surgery. However, incorporation of a network in control loops results in various constraints such as time delays and packet dropouts, due to limited bandwidth, quantization errors caused by hybrid nature of NCSs, variable sampling or transmission intervals due to multiple nodes, clock asynchronization among local and remote nodes, network security and safety, and network security due to shared communication networks [6-8]. It is generally known that any of these networked-induced communication imperfections and constraints can degrade closed-loop performance or, even worse, can harm closed-loop stability of NCSs. Therefore, it is important to know how these effects influence the stability properties.

Recently, systematic approaches to analyze stability of NCSs subject to only one of these networked-induced imperfections are well developed. For instance, the effects of packet dropouts are studied in [9-14], and of time delays in [15-24], of quantization in [7, 25-28], of time-varying transmission intervals and communication constraints in [29-31] and [32-35], respectively. However, in NCSs all the aforementioned limitations and constraints are present simultaneously. Unfortunately, most of the results are available that study single problem of these imperfections. In this paper, we will 
focus on the modeling and stability of NCSs with random time delays and packet dropouts.

In fact, packet dropout and time delay are both important issues that could severely destabilize NCSs. To study these issues, much research has been done on the effect of packet dropout and time delay on NCSs. As to the packet dropout, many approaches have been developed. Stabilization problem of NCSs with both arbitrary and Markovian packet losses is considered in [9]. The $H_{\infty}$ control problem for NCSs with packet dropouts is studied in $[10,13]$. A new NCSs model is considered for NCSs with single- and multiple-packet transmissions in [11]. The control problem for NCSs with Bernoulli-distributed stochastic packet losses is revisited in [14]. As to the time delay, great efforts have been made on stability analysis and controller design for NCSs with interval time-varying delay, uncertain time delay, large time delay, or random time delay, as presented in [15-23] (for more details, please refer to the literature therein).

So far, most of the results considered the networkinduced delay and data packet loss separately, while in practice, the time delay and packet dropout are present simultaneously in NCSs. Fewer results are available that study combinations of time delay and packet dropout in the literature [36-42]. Furthermore, in [36-38], the switched system method is studied for NCSs with both delay and packet dropout. The two-mode-dependent state feedback controller design for NCSs with time delays and packet dropouts is addressed by augmenting the state variable approach in [39]. In [40] the robust $H_{\infty}$ filtering problem for a class of uncertain nonlinear networked systems with both stochastic time-varying communication delays and packet dropouts is investigated. In [41] the problem of $H_{\infty}$ controller design for NCSs with time delay and packet dropout by applying the linear estimation-based time delay and packet dropout compensation method is considered. The receding horizon $H_{\infty}$ control problem for a class of NCSs with random delay and packet disordering is investigated by using the receding optimization principle in [42]. To the best of the authors' knowledge, up to now, little attention has been paid to the study of unified time delays and packet dropouts model and output feedback controller design for NCSs based on Markovian jump linear system (MJLS) with Markovian time delays model, which motivates the study of this paper.

In this paper, we address the unified model, stability analysis, and output feedback controller design of NCSs with the sensor-to-controller (S-C) and controller-to-actuator (C-A) random time delays and packet dropouts under an MJLS [43] framework. The present paper involves three contributions compared to the previous relevant works. The first is in the unified new model of NCSs with random network time delays and packet dropouts. The second one is that the Lyapunov functional method is used for stochastic stability analysis for NCSs. Compared with the extended matrix method, the Lyapunov functional avoids augmenting the dimension of the closed-loop system state, thus avoiding the complicated computation and conservatism caused by extended matrix method. The third one is the output feedback controller which is used to stabilize the closed-loop system, and the

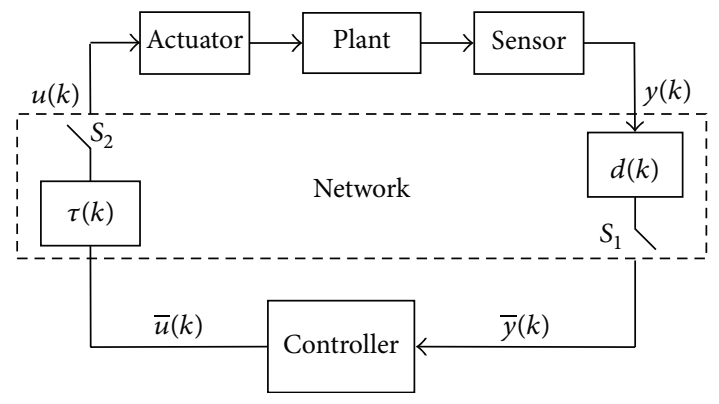

FIGURE 1: The structure of the NCS with random delays and/or packet dropouts.

results are applied to a classical angular positioning system to illustrate the effectiveness of the approach.

Notations. In the sequel, matrices are assumed to have appropriate dimensions. $\mathbb{R}^{n}$ and $\mathbb{R}^{n \times m}$ denote, respectively, the $n$ dimensional Euclidean space and the set of all $n \times m$ real matrices. The notations $A>0$ and $A<0$ are used to denote a positive and negative definite matrices, respectively. $\operatorname{diag}\left(A_{1}, \ldots, A_{n}\right)$ refers to an $n \times n$ diagonal matrix with $A_{i}$ as its $i$ th diagonal entry. $I$ and 0 denote identity matrix and zero matrix with appropriate dimensions, respectively. The superscript ${ }^{T}$ denotes the transpose for vectors or matrices. $\mathbb{E}[\cdot]$ denotes the mathematical expectation operator. The symbol $*$ within a matrix represents the symmetric entries.

\section{Problem Description}

In this paper, we consider the following linear discrete-time system:

$$
\begin{gathered}
x_{p}((k+1) T)=A x_{p}(k T)+B u(k T), \\
y(k T)=C x_{p}(k T)
\end{gathered}
$$

where $x_{p}(k T) \in \mathbb{R}^{n}$ is the system state, $u(k T) \in \mathbb{R}^{m}$ is the control input, and $y(k T) \in \mathbb{R}^{p}$ is the measurable output. $k T$ and $T$ are the sampling instant and the sampling period of the sensor. $A, B$, and $C$ are known real constant matrices with appropriate dimensions. In this paper, for simplicity of presentation, $k T$ is denoted by $k$.

Considering the same assumption in [16], the sensor, the controller, and the actuator are time driven and are connected over a network medium. The framework of the system over a network medium is depicted in Figure 1. The holder is chosen as zero-order hold $(\mathrm{ZOH})$ holder. Under these assumptions, it is known that the controller and actuator update at the instant $k$ only will always use the most recent data; otherwise, it will maintain the old data. In the NCS as in Figure 1, network-induced time delays and packet dropouts exist in the communication links between the S-C and C-A.

Random time delays exist in the S-C and C-A, as shown in Figure 1. Random but bounded scalar $d(k) \geq 0$ and $\tau(k) \geq 0$ are the random S-C and C-A time delays, respectively. It is assumed that both $d(k)$ and $\tau(k)$ are bounded, which are $0 \leq \underline{d} \leq d(k) \leq \bar{d}, 0 \leq \underline{\tau} \leq \tau(k) \leq \bar{\tau}$, where $\underline{d}=\min \{d(k)\}$, 
$\bar{d}=\max \{d(k)\}, \underline{\tau}=\min \{\tau(k)\}$, and $\bar{\tau}=\max \{\tau(k)\}$. One way to model the delays $d(k)$ and $\tau(k)$ is using the finite state Markov chains as shown in $[19,44-46]$. The main advantages of the Markov model is as follows: the dependency between delays is taken into account since in real networks the current time delays are usually related with the previous delays [45]. In this paper $d(k)$ and $\tau(k)$ are modeled as two homogeneous Markov chains that take value in $\bar{S}_{2}=\left\{0,1, \ldots, s_{2}\right\}$ and $\bar{S}_{3}=\left\{0,1, \ldots, s_{3}\right\} \cdot \lambda=\left\{\lambda_{m n}\right\}$ and $\gamma=\left\{\gamma_{q p}\right\}$ denote transition probability matrices of $d(k)$ and $\tau(k)$, respectively, with probabilities $\lambda_{m n}$ and $\pi_{i j}$, which are defined in (7).

In Figure $1, S_{1}$ and $S_{2}$ denote the network switches between the $\mathrm{S}-\mathrm{C}$ and $\mathrm{C}-\mathrm{A}$, respectively. $\alpha$ and $\beta(\alpha=0,1 ; \beta=$ $0,1)$ denote the states of $S_{1}$ and $S_{2}$. When $S_{1}\left(S_{2}\right)$ is in state $\alpha=0(\beta=0)$, the packet is received successfully and the $\bar{y}(k)=y(k-d(k))(u(k)=\bar{u}(k-\tau(k)))$. Whereas when $S_{1}\left(S_{2}\right)$ is in state $\alpha=1(\beta=1)$, the packet is lost and the switch output (control input) is held at the previous value $\bar{y}(k)=\bar{y}(k-1)(u(k)=u(k-1))$. The behavior of the S-C and C-A time delays and packet dropouts can be modeled as

$$
\begin{aligned}
& \bar{y}(k)=(1-\alpha) y(k-d(k))+\alpha \bar{y}(k-1), \\
& u(k)=(1-\beta) \bar{u}(k-\tau(k))+\beta u(k-1),
\end{aligned}
$$

where

$$
\begin{gathered}
\alpha= \begin{cases}0, & \text { if } S_{1} \text { is closed and the packet is received } \\
1, & \text { otherwise, }\end{cases} \\
\beta= \begin{cases}0, & \text { if } S_{2} \text { is closed the packet is received } \\
1, & \text { otherwise. }\end{cases}
\end{gathered}
$$

From the above analysis, the random data packet dropouts in $\mathrm{S}-\mathrm{C}$ and $\mathrm{C}-\mathrm{A}$ can be modeled a discrete-time homogeneous Markov chain $\mu(k)$ with four modes. Four modes of Markov chain $\mu(k)=1, \mu(k)=2, \mu(k)=3$, and $\mu(k)=4$ are corresponding to four states $(\alpha=0, \beta=0),(\alpha=0, \beta=1)$, $(\alpha=1, \beta=0)$, and $(\alpha=1, \beta=1)$ of network switches $S_{1}$ and $S_{2}$.

This paper studies the stability of system (1) under an output feedback controller; that is,

$$
\bar{u}(k)=K \bar{y}(k)
$$

where $K$ is the output feedback controller gain.

Remark 1. There are two ways to design the controller (4). One is adopting a mode-independent controller, and another one is using a mode-dependent controller (the controller gain dependent on the information of time delays and packet losses). However, the mode-independent controller (4) can avoid the controller gain switching in high frequency following the multimodes of Markov chains $\mu(k), \tau(k)$, and $d(k)$ in system (5). Hence, in this paper, we choose the modeindependent controller.

Let $x(k)=\left[x_{p}^{T}(k) \bar{y}^{T}(k-1) u^{T}(k-1)\right]^{T}$ be the augmented state vector. Applying the controller (4) to (1) and (2), we can obtain the closed-loop system for the NCS with random time delays and packet dropouts in Figure 1 as

$$
\begin{gathered}
x(k+1)=\bar{A}(\mu(k)) x(k)+\sum_{s=1}^{3} \bar{B}_{s}(\mu(k)) H_{s} x\left(k-\eta_{s}(k)\right), \\
x(k)=\varphi(k), \quad k=-\bar{d}-\bar{\tau},-\bar{d}-\bar{\tau}+1, \ldots, 0,
\end{gathered}
$$

where

$$
\begin{aligned}
& \bar{A}(\mu(k))=\left[\begin{array}{ccc}
A & 0 & \beta B \\
0 & \alpha I & 0 \\
0 & 0 & \beta I
\end{array}\right], \\
& \bar{B}_{1}(\mu(k))=\left[\begin{array}{c}
0 \\
(1-\alpha) C \\
0
\end{array}\right] \text {, } \\
& \bar{B}_{2}(\mu(k))=\widetilde{B}_{2}(\mu(k)) K, \\
& \bar{B}_{3}(\mu(k))=\widetilde{B}_{3}(\mu(k)) K C, \\
& \widetilde{B}_{2}(\mu(k))=\left[\begin{array}{c}
\alpha(1-\beta) B \\
0 \\
\alpha(1-\beta) I
\end{array}\right], \\
& \widetilde{B}_{3}(\mu(k))=\left[\begin{array}{c}
(1-\alpha)(1-\beta) B \\
0 \\
(1-\alpha)(1-\beta) I
\end{array}\right] \text {, } \\
& H_{1}=\left[\begin{array}{lll}
I & 0 & 0
\end{array}\right], \quad H_{2}=\left[\begin{array}{lll}
0 & I & 0
\end{array}\right] \text {, } \\
& H_{3}=\left[\begin{array}{lll}
I & 0 & 0
\end{array}\right] \text {, } \\
& \eta_{1}(k)=d(k), \quad \eta_{2}(k)=\tau(k), \\
& \eta_{3}(k)=d(k-\tau(k))+\tau(k) \approx d(k)+\tau(k), \\
& \bar{d}=\max \{d(k)\}, \quad \underline{d}=\min \{d(k)\}, \\
& \bar{\tau}=\max \{\tau(k)\}, \quad \underline{\tau}=\min \{\tau(k)\},
\end{aligned}
$$

$\varphi(k), k=-\bar{d}-\bar{\tau},-\bar{d}-\bar{\tau}+1, \ldots, 0$ is the system initial value.

Remark 2. In (5), we assume that delay steps $\eta_{3}(k)=d(k-$ $\tau(k))+\tau(k) \approx d(k)+\tau(k)$. There are three reasons to support the ideal hypothesis: the first one is that $d(k)$ and $d(k-\tau(k))$ take values in the same finite set $\bar{S}_{2}=\left\{0,1, \ldots, d_{\text {max }}\right\}$; the second one is that $\eta_{3}(k)=d(k-\tau(k))+\tau(k)$ and $d(k)+\tau(k)$ take values in the same finite set $\left\{0,1,2, \ldots, d_{\max }+\tau_{\max }\right\}$; and the third one is that for system $(5), \eta_{3}(k)$ denotes the delay steps of system state $x(k)$; using $(d(k)+\tau(k))$ approximation equal to $(d(k-\tau(k))+\tau(k))$ will not affect the performance of system (5). Therefore, $\eta_{3}(k)=d(k-\tau(k))+\tau(k)$ will be replaced by $\eta_{3}(k)=d(k)+\tau(k)$ in the following.

In system (5), $\{\mu(k), k \in \mathbb{Z}\},\{d(k), k \in \mathbb{Z}\}$ and $\{\tau(k), k \in$ $\mathbb{Z}\}$, are three finite state discrete-time homogeneous Markov chains. The three Markov chains take values in the finite sets 
$\bar{S}_{1}=\left\{1,2, \ldots, s_{1}\right\}, \bar{S}_{2}=\left\{0,1, \ldots, s_{2}\right\}$, and $\bar{S}_{3}=\left\{0,1, \ldots, s_{3}\right\}$ with transition probabilities

$$
\begin{array}{cl}
\operatorname{Pr}\{\mu(k+1)=j \mid \mu(k)=i\}=\pi_{i j}, & \pi_{i}=\operatorname{Pr}\left(\pi_{0}=i\right), \\
\operatorname{Pr}\{d(k+1)=n \mid d(k)=m\}=\lambda_{m n}, & \lambda_{m}=\operatorname{Pr}\left(\lambda_{0}=m\right), \\
\operatorname{Pr}\{\tau(k+1)=q \mid \tau(k)=p\}=\gamma_{p q}, & \gamma_{p}=\operatorname{Pr}\left(\gamma_{0}=p\right),
\end{array}
$$

where $\pi_{i j} \geq 0, \lambda_{m n} \geq 0$, and $\gamma_{p q} \geq 0$ for all $i, j \in \bar{S}_{1}, m, n \in \bar{S}_{2}$, $p, q \in \bar{S}_{3}$ and

$$
\begin{gathered}
\sum_{j=1}^{s_{1}} \pi_{i j}=1, \quad \sum_{n=0}^{s_{2}} \lambda_{m n}=1, \\
\sum_{q=0}^{s_{3}} \gamma_{p q}=1 .
\end{gathered}
$$

For $\mu(k)=i, i \in \bar{S}_{1}$, when $i=1, i=2, i=3$, and $i=4$, the $\alpha$ and $\beta$ in (5) take values $(\alpha=0, \beta=0)$, $(\alpha=0, \beta=1),(\alpha=1, \beta=0)$, and $(\alpha=1, \beta=1)$, respectively. $\bar{A}(\mu(k)), \bar{B}_{1}(\mu(k)), \widetilde{B}_{2}(\mu(k))$, and $\widetilde{B}_{3}(\mu(k))$ are known constant matrices of appropriate dimensions.

Remark 3. The closed-loop system (5) is a Markovian jump linear system with multiple Markov chains, which describe the behavior of the S-C and C-A time delays and packet dropouts, respectively. This enables us to analyze and synthesize such NCSs by applying Markovian jump linear system theory. Note that the problem of unified modeling and output feedback control for NCSs with both S-C and C-A time delays and packet dropouts modeled by multiple Markov chains has not been done in the literature.

Definition 4 (see $[11,47])$. System (5) is stochastically stable if there exists a constant $C$ such that

$$
\begin{array}{r}
\sum_{k=0}^{\infty} \mathbb{E}\left[\|x(k)\|^{2} \mid x_{0}, \mu_{0}, d_{0}, \tau_{0}\right] \\
\leq C Y\left(x_{0}, \varphi_{i},-d_{\max }-\tau_{\max }\right. \\
\left.\leq i \leq 0, \mu_{0}, d_{0}, \tau_{0}\right)
\end{array}
$$

where $\Upsilon\left(x_{0}, \varphi_{i},-\bar{d}-\bar{\tau} \leq i \leq 0, \mu_{0}, d_{0}, \tau_{0}\right)$ is a nonnegative function and the system initial values satisfy $\Upsilon(0,0, \ldots, 0)=0$.

\section{Main Results}

By applying a new Lyapunov functional, sufficient conditions for the stochastic stability, the synthesis of controller design for system (5) will be established in this section.

Theorem 5. For system (5), given random but bounded scalar $d(k) \in[\underline{d} \bar{d}]$ and $\tau(k) \in[\underline{\tau} \bar{\tau}]$, if for each mode $i \in \bar{S}_{1}, m \in \bar{S}_{2}$, and $p \in \bar{S}_{3}$, there exist matrices $P_{i, m, p}>0, Q_{1 s}>0, Q_{2 s}>0$,
$Q_{3 s}>0, R_{1 s}>0$, and $R_{2 s}>0, s=1,2,3$, such that the following matrix inequalities:

$$
\left[\begin{array}{cccc}
\Xi_{1} & \Xi_{2} & \Xi_{3} & \Xi_{4} \\
* & -\bar{P}_{i, m, p} & 0 & 0 \\
* & * & -\bar{R}_{1} & 0 \\
* & * & * & -\bar{R}_{2}
\end{array}\right]<0,
$$

where

$$
\begin{aligned}
& \Xi_{1}=\left[\begin{array}{cccc}
\Pi_{i, m, p} & \Omega_{12} & 0 & 0 \\
* & -\bar{Q}_{3}-\Omega_{22}-2 \Psi & \Psi & \Omega_{24}+\Psi \\
* & * & -\bar{Q}_{2}-\Psi & 0 \\
* & * & * & -\bar{Q}_{1}-\Omega_{44}-\Psi
\end{array}\right] \\
& \Xi_{2}=\left[\begin{array}{lllll}
\bar{P}_{i, m, p} \bar{A}_{i} & \bar{P}_{i, m, p} \Lambda & 0 & 0
\end{array}\right]^{T}, \\
& \Xi_{3}=\left[\begin{array}{lll}
\Xi_{31} & \Xi_{32} & \Xi_{33}
\end{array}\right], \quad \Xi_{4}=\left[\begin{array}{lll}
\Xi_{41} & \Xi_{42} & \Xi_{43}
\end{array}\right], \\
& \Xi_{31}=\left[\begin{array}{llll}
\bar{t}_{1} R_{11} H_{1}\left(\bar{A}_{i}-I\right) & \bar{t}_{1} R_{11} \bar{\Lambda} & 0 & 0
\end{array}\right]^{T}, \\
& \Xi_{32}=\left[\begin{array}{llll}
\bar{t}_{2} R_{12} H_{2}\left(\bar{A}_{i}-I\right) & \bar{t}_{2} R_{12} \bar{\Lambda} & 0 & 0
\end{array}\right]^{T}, k \\
& \Xi_{33}=\left[\begin{array}{llll}
\bar{t}_{3} R_{13} H_{3}\left(\bar{A}_{i}-I\right) & \bar{t}_{3} R_{13} \bar{\Lambda} & 0 & 0
\end{array}\right]^{T} \text {, }
\end{aligned}
$$

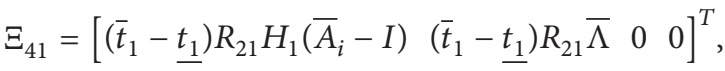

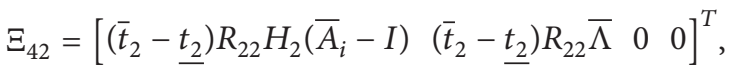

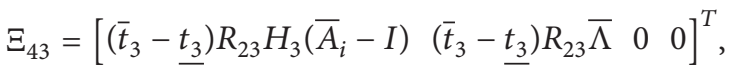

$$
\begin{aligned}
& \bar{P}_{i, m, p}=\sum_{j=1}^{s_{1}} \sum_{n=0}^{s_{2}} \sum_{q=0}^{s_{3}} \pi_{i j} \lambda_{m n} \gamma_{p q} P_{j, n, q},
\end{aligned}
$$

$$
\begin{aligned}
\bar{R}_{1}=\operatorname{diag} & \left\{R_{11}, R_{12}, R_{13}\right\}, \quad \bar{R}_{2}=\operatorname{diag}\left\{R_{21}, R_{22}, R_{23}\right\}, \\
\Pi_{i, m, p}= & -P_{i, m, p} \\
& +\sum_{s=1}^{3}\left[H_{s}^{T}\left(Q_{1 s}+Q_{2 s}+\left(\bar{t}_{s}-\underline{t_{s}}+1\right) Q_{3 s}\right) H_{s}\right] \\
& -\Omega_{11},
\end{aligned}
$$

$$
\begin{aligned}
& \Omega_{11}=\sum_{s=1}^{3} H_{s}^{T} R_{1 s} H_{s}, \quad \Omega_{12}=\left[\begin{array}{lll}
H_{1}^{T} R_{11} & H_{2}^{T} R_{12} & H_{3}^{T} R_{13}
\end{array}\right], \\
& \Omega_{22}=\operatorname{diag}\left\{2 R_{11}, 2 R_{12}, 2 R_{13}\right\}, \\
& \Omega_{24}=\Omega_{44}=\operatorname{diag}\left\{R_{11}, R_{12}, R_{13}\right\} \text {, } \\
& \Psi=\operatorname{diag}\left\{R_{21}, R_{22}, R_{23}\right\}, \quad \bar{Q}_{1}=\operatorname{diag}\left\{Q_{11}, Q_{12}, Q_{13}\right\}, \\
& \bar{Q}_{2}=\operatorname{diag}\left\{Q_{21}, Q_{22}, Q_{23}\right\}, \quad \bar{Q}_{3}=\operatorname{diag}\left\{Q_{31}, Q_{32}, Q_{33}\right\} \text {, } \\
& \Lambda=\left[\begin{array}{lll}
\bar{B}_{i 1} & \bar{B}_{i 2} & \bar{B}_{i 3}
\end{array}\right], \quad \bar{\Lambda}=\left[\begin{array}{lll}
H_{1} \bar{B}_{i 1} & H_{2} \bar{B}_{i 2} & H_{3} \bar{B}_{i 3}
\end{array}\right], \\
& \bar{A}_{i}=\bar{A}(\mu(k)), \quad \bar{B}_{i 1}=\bar{B}_{1}(\mu(k)),
\end{aligned}
$$




$$
\begin{gathered}
\bar{B}_{i 2}=\bar{B}_{2}(\mu(k)), \\
\bar{B}_{i 3}=\bar{B}_{3}(\mu(k)), \\
\bar{t}_{1}=\bar{d}, \quad \underline{t_{1}}=\underline{d}, \quad \bar{t}_{2}=\bar{\tau}, \quad \underline{t_{2}}=\underline{\tau}, \\
\bar{t}_{3}=\bar{d}+\bar{\tau}, \quad \underline{t_{3}}=\underline{d}+\underline{\tau},
\end{gathered}
$$

$\bar{A}(\mu(k)), \bar{B}_{1}(\mu(k)), \bar{B}_{2}(\mu(k))$, and $\bar{B}_{3}(\mu(k))$ defined in (5) hold for all $i, j \in \bar{S}_{1}, m, n \in \bar{S}_{2}$, and $p, q \in \bar{S}_{3}$, then system (5) is stochastically stable.

Proof. It is given in the appendix.

Theorem 5 gives a sufficient condition for the stochastic stability of system (5). However, it should be noted that the conditions (10) are no more LMI conditions. To handle this, the equivalent LMI conditions are given in Theorem 6 by cone complementarity linearization (CCL) algorithm.

Theorem 6. Consider system (5) with random but bounded scalar $d(k) \in[\underline{d} \bar{d}]$ and $\tau(k) \in[\underline{\tau} \bar{\tau}]$. There exists an output feedback controller (4) such that the resulting closed-loop system is stochastically stable if for each mode $i \in \bar{S}_{1}, m \in \bar{S}_{2}$, and $p \in \bar{S}_{3}$, there exist matrices $P_{i, m, p}>0, X_{i, m, p}>0, Q_{1 s}>0$, $Q_{2 s}>0, Q_{3 s}>0, R_{1 s}>0, R_{2 s}>0, U_{1 s}>0$, and $U_{2 s}>0$, $s=1,2,3$ and $K$ such that

$$
\begin{gathered}
{\left[\begin{array}{cccc}
\Xi_{1} & \widehat{\Xi}_{2} & \widehat{\Xi}_{3} & \widehat{\Xi}_{4} \\
* & -X_{j, n, q} & 0 & 0 \\
* & * & -U_{1 s} & 0 \\
* & * & * & -U_{2 s}
\end{array}\right]<0,} \\
P_{i, m, p} X_{i, m, p}=I, \quad R_{1 s} U_{1 s}=I, \quad R_{2 s} U_{2 s}=I, \\
s=1,2,3,
\end{gathered}
$$

where

$$
\begin{aligned}
& \widehat{\Xi}_{2}=\left[\begin{array}{lllll}
\mathscr{L}_{i, m, p} \bar{A}_{i} & \mathscr{L}_{i, m, p} \Lambda & 0 & 0
\end{array}\right]^{T}, \\
& \widehat{\Xi}_{3}=\left[\begin{array}{lll}
\widehat{\Xi}_{31} & \widehat{\Xi}_{32} & \widehat{\Xi}_{33}
\end{array}\right], \quad \widehat{\Xi}_{4}=\left[\begin{array}{lll}
\widehat{\Xi}_{41} & \widehat{\Xi}_{42} & \widehat{\Xi}_{43}
\end{array}\right], \\
& \widehat{\Xi}_{31}=\left[\begin{array}{llll}
\bar{t}_{1} H_{1}\left(\bar{A}_{i}-I\right) & \bar{t}_{1} \bar{\Lambda} & 0 & 0
\end{array}\right]^{T}, \\
& \widehat{\Xi}_{32}=\left[\begin{array}{llll}
\bar{t}_{2} H_{2}\left(\bar{A}_{i}-I\right) & \bar{t}_{2} \bar{\Lambda} & 0 & 0
\end{array}\right]^{T} \text {, } \\
& \widehat{\Xi}_{33}=\left[\begin{array}{llll}
\bar{t}_{3} H_{3}\left(\bar{A}_{i}-I\right) & \bar{t}_{3} \bar{\Lambda} & 0 & 0
\end{array}\right]^{T} \text {, } \\
& \widehat{\Xi}_{41}=\left[\begin{array}{lll}
\left(\bar{t}_{1}-\underline{t_{1}}\right) H_{1}\left(\bar{A}_{i}-I\right)\left(\bar{t}_{1}-\underline{t_{1}}\right) \bar{\Lambda} & 0 & 0
\end{array}\right]^{T} \text {, }
\end{aligned}
$$

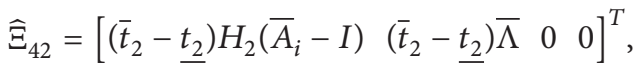

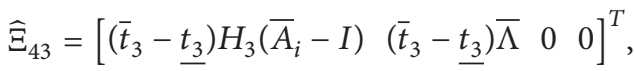

$$
\begin{aligned}
& X_{j, n, q}=\operatorname{diag} \underbrace{\left\{X_{1,0,0}, X_{1,0,1}, \ldots, X_{j, n, q}, \ldots, X_{s_{1}, s_{2}, s_{3}}\right\}} \\
& \left(s_{1}\left(1+s_{2}\right)\left(1+s_{3}\right)\right) \text { items }
\end{aligned}
$$

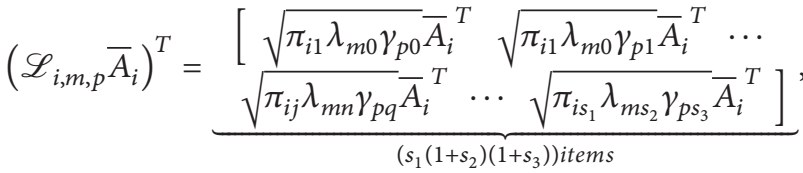

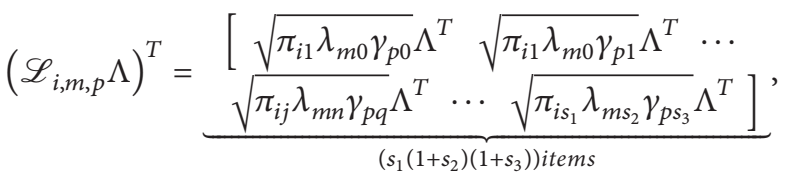

$\Xi_{1}, \bar{A}_{i}, \Lambda$, and $\bar{\Lambda}$ are defined in Theorem 5. Moreover, if (12) and (13) have solutions, the controller gain is given by $K$.

Proof. By Schur complement, (12) is equivalent to

$$
\left[\begin{array}{cccc}
\Xi_{1} & \widehat{\Xi}_{2} & \widehat{\Xi}_{3} & \widehat{\Xi}_{4} \\
* & -P_{j, n, q}^{-1} & 0 & 0 \\
* & * & -R_{1 s}^{-1} & 0 \\
* & * & * & -R_{2 s}^{-1}
\end{array}\right]<0, \quad s=1,2,3 .
$$

Let $P_{j, n, q}^{-1}=X_{j, n, q}, R_{1 s}^{-1}=U_{1 s}$, and $R_{2 s}^{-1}=U_{2 s}$; then we can obtain (12) and (13). This completes the proof.

The conditions in Theorem 6 are a set of LMIs with some matrix inverse constraints. Although they are nonconvex, which prevents us from solving them using the existing convex optimization tool, we can use the CCL algorithm to transform this problem into the nonlinear minimization problem with LMI constraints as follows:

$$
\min \operatorname{Trace}\left(\sum_{s=1}^{3}\left(R_{1 s} U_{1 s}+R_{2 s} U_{2 s}\right)+\sum_{j=1}^{s_{1}} \sum_{n=0}^{s_{2}} \sum_{q=0}^{s_{3}} P_{i, m, p} X_{i, m, p}\right)
$$

$$
\text { s.t. }\left\{\begin{array}{l}
\text { (i) }(12) \\
\text { (ii) }\left[\begin{array}{cc}
R_{1 s} & I \\
* & U_{1 s}
\end{array}\right]>0, \quad s=\{1,2,3\}, \\
\text { (iii) }\left[\begin{array}{cc}
R_{2 s} & I \\
* & U_{2 s}
\end{array}\right]>0, \quad s=\{1,2,3\}, \\
\text { (iv) }\left[\begin{array}{cc}
P_{i, m, p} & I \\
* & X_{i, m, p}
\end{array}\right]>0, \quad i \in \bar{S}_{1}, m \in \bar{S}_{2}, p \in \bar{S}_{3} .
\end{array}\right.
$$

The above nonlinear minimization problem can be solved by the CCL algorithm presented in the following.

Algorithm 7. Consider the following steps.

Step 1. Find a feasible solution satisfying LMIs (i), (ii), (iii), and (iv) in (17), set as $\left(R_{1 s}^{0}, U_{1 s}^{0}, R_{2 s}^{0}, U_{2 s}^{0}, P_{i, m, p}^{0}, X_{i, m, p}^{0}\right), s=$ $\{1,2,3\}$ and $k=0$.

Step 2. Solve the following LMI optimization problem for variables $\left(R_{1 s}, U_{1 s}, R_{2 s}, U_{2 s}, s=\{1,2,3\}, P_{i, m, p}, X_{i, m, p}, K^{0}\right)$ : Minimize trace $\left\{\sum_{s=1}^{3}\left(\left(R_{1 s}^{k} U_{1 s}+R_{1 s} U_{1 s}^{k}\right)+\left(R_{2 s}^{k} U_{2 s}+R_{2 s} U_{2 s}^{k}\right)\right)+\right.$ $\left.\sum_{j=1}^{s_{1}} \sum_{n=0}^{s_{2}} \sum_{q=0}^{s_{3}}\left(P_{i, m, p}^{k} X_{i, m, p}+P_{i, m} X_{i, m, p}^{k}\right)\right\}$ subject to LMIs (17). 


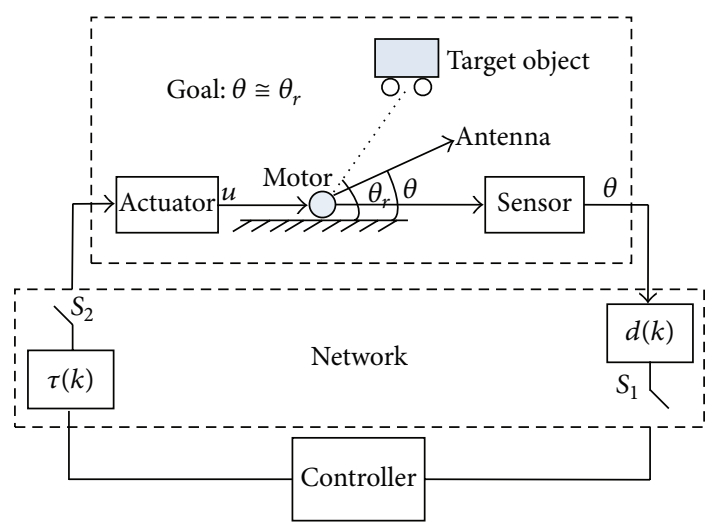

Figure 2: The angular positioning system.

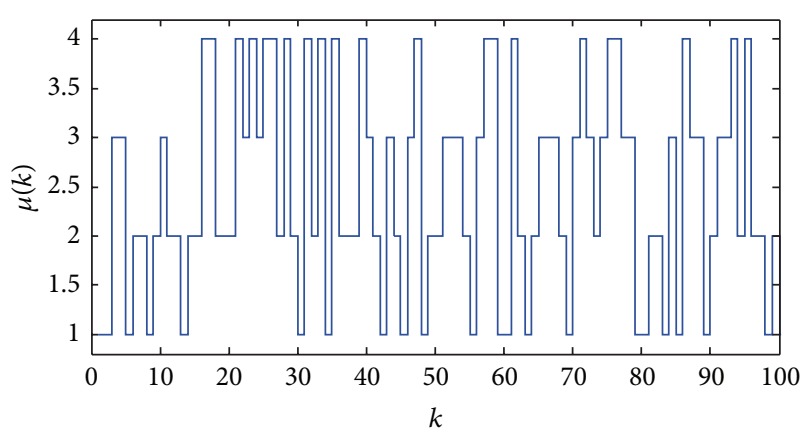

Figure 3: Values of $\mu(k)$.

Set $R_{1 s}^{k+1}=R_{1 s}, U_{1 s}^{k+1}=U_{1 s}, R_{2 s}^{k+1}=R_{2 s}, U_{2 s}^{k+1}=U_{2 s}, s=\{1,2$, 3\}, $P_{i, m, p}^{k+1}=P_{i, m, p}, X_{i, m, p}^{k+1}=X_{i, m, p}$, and $K^{k+1}=K$.

Step 3. If LMI (i) in (17) is satisfied, then exit the iteration. If LMI (i) in (17) is not satisfied, let $k=k+1$ and then return to Step 2.

\section{Numerical Example}

In this section, a simulation example is given to illustrate the usefulness of the developed method. Consider a classical angular positioning system $[42,48,49]$ in Figure 2, which consists of a rotating antenna at the origin of the plane, driven by an electric motor. Assume that the angular position of the antenna $\theta$ (rad), the angular position of the moving object $\theta_{r}(\mathrm{rad})$, and the angular velocity of the antenna $\dot{\theta}\left(\mathrm{rad} \mathrm{s}^{-1}\right)$ are measurable. The state variables are chosen as $[\theta \dot{\theta}]^{T}$ and the output is $y=\theta^{T}$. The control problem is to use the input voltage to the motor to rotate the antenna so that it always point in the direction of a moving object in the plant. The output feedback controller is designed for the following values of the matrices $A, B$, and $C$ :

$$
\begin{gathered}
A=\left[\begin{array}{cc}
1 & 0.0995 \\
0 & 0.99
\end{array}\right], \quad B=\left[\begin{array}{l}
0.0039 \\
0.0783
\end{array}\right], \\
C=\left[\begin{array}{cc}
1.4 & 0.8 \\
-0.2 & 0.4
\end{array}\right] .
\end{gathered}
$$

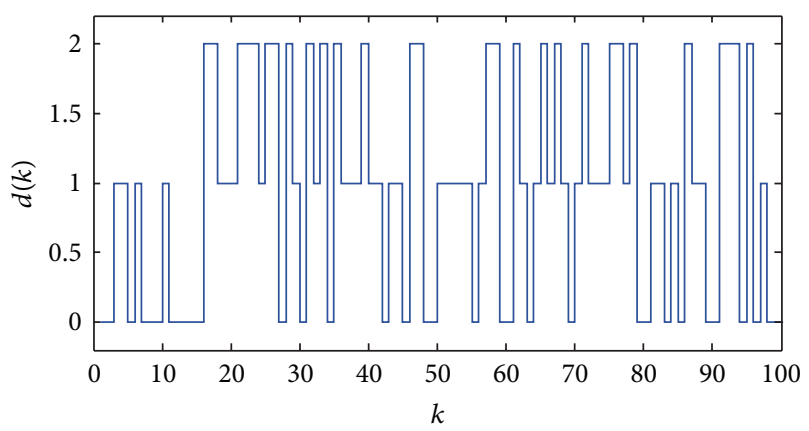

Figure 4: S-C random delays $d(k)$.

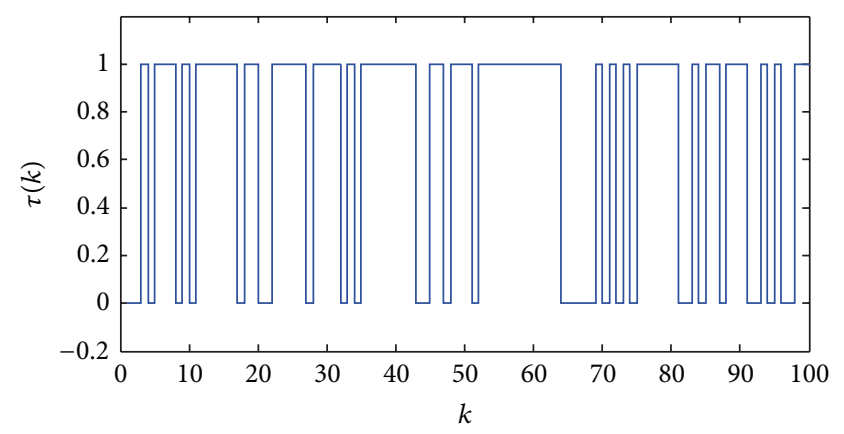

FIGURE 5: C-A random delays $\tau(k)$.

The stochastic jumping parameter $\mu(k) \in\{1,2,3,4\}$ and the random delays involved in system $(5)$ which are $d(k)=m$, $m \in\{0,1,2\}$ and $\tau(k)=p, p \in\{0,1\}$, and their transition matrices are given by

$$
\begin{gathered}
\pi=\left[\begin{array}{llll}
0.4 & 0.3 & 0.2 & 0.1 \\
0.2 & 0.5 & 0.2 & 0.1 \\
0.3 & 0.2 & 0.4 & 0.1 \\
0.1 & 0.2 & 0.2 & 0.5
\end{array}\right], \quad \lambda=\left[\begin{array}{lll}
0.5 & 0.2 & 0.3 \\
0.4 & 0.5 & 0.1 \\
0.3 & 0.2 & 0.5
\end{array}\right], \\
\gamma=\left[\begin{array}{ll}
0.5 & 0.5 \\
0.8 & 0.2
\end{array}\right] .
\end{gathered}
$$

Figures 3, 4, and 5 show part of the simulation of the stochastic jumping parameter $\mu(k)$, S-C delay $d(k)$, and CA delay $\tau(k)$ governed by their corresponding transition probability matrices, respectively.

The initial value $x(0)=\left[\begin{array}{ll}0.01 & 0\end{array}\right]^{T}$. By Theorem 6, we can obtain the gain matrices $K$ of controller (4) are constructed as $K=[-0.0088-0.0117]$.

The state trajectories are shown in Figure 6, where two curves represent state trajectories under the controller gains $K$ and Figure 6 indicates that system (5) is stochastically stable.

\section{Conclusion}

The output feedback stabilization problem for NCSs with both S-C and C-A random time delays and packet dropouts is investigated in this paper. The $\mathrm{S}-\mathrm{C}$ and $\mathrm{C}-\mathrm{A}$ random time delays and packet dropouts are modeled by independent 


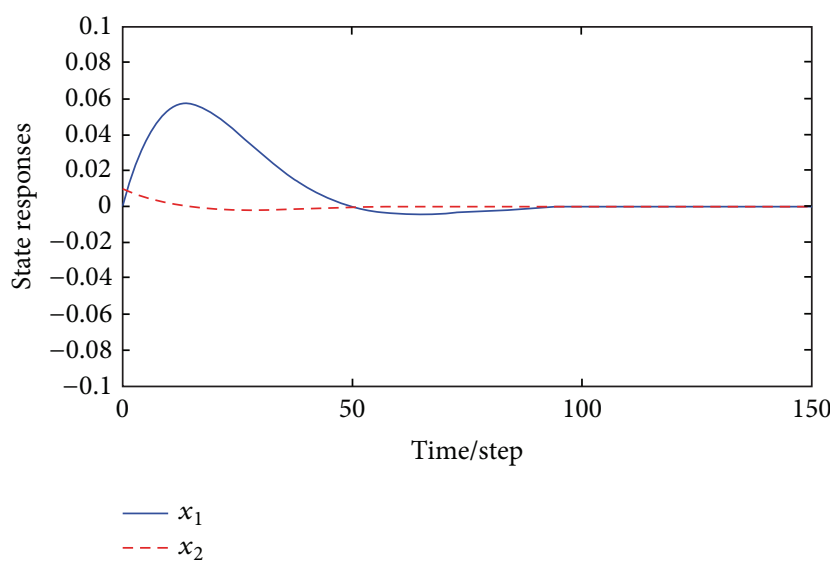

FIGURE 6: State trajectories under $K$.

multiple Markov chains. Then the resulting closed-loop system is modeled as a Markovian jump linear system with Markov delays. Sufficient conditions on stochastic stability and stabilization are obtained by Lyapunov stability theory and linear matrix inequalities method. The CCL algorithm is employed to obtain the output feedback controller. Finally, an example is presented to illustrate the effectiveness of the approach.

\section{Appendix}

\section{A. Proof of Theorem 5}

Proof. For the closed-loop system (5), construct a LyapunovKrasovskii functional as

$$
V(k)=V_{1}(k)+V_{2}(k)+V_{3}(k)+V_{4}(k),
$$

where

$$
\begin{aligned}
V_{1}(k)= & x(k)^{T} P(\mu(k), d(k), \tau(k)) x(k), \\
V_{2}(k)= & \sum_{s=1}^{3} \sum_{l=k-\bar{t}_{s}}^{k-1} x(l)^{T} H_{s}^{T} Q_{1 s} H_{s} x(l) \\
& +\sum_{s=1}^{3} \sum_{l=k-t_{s}}^{k-1} x(l)^{T} H_{s}^{T} Q_{2 s} H_{s} x(l), \\
V_{3}(k)= & \sum_{s=1}^{3} \sum_{\theta=-\bar{\eta}_{s}+2}^{-\eta_{s}+1} \sum_{l=k+\theta-1}^{k-1} x(l)^{T} H_{s}^{T} Q_{3 s} H_{s} x(l) \\
& +\sum_{s=1}^{3} \sum_{l=k-\eta_{s}(k)}^{k-1} x(l)^{T} H_{s}^{T} Q_{3 s} H_{s} x(l)
\end{aligned}
$$

$$
\begin{aligned}
V_{4}(k)= & \sum_{s=1}^{3} \sum_{\theta=-\bar{\eta}_{s}+1}^{0} \sum_{l=k+\theta-1}^{k-1} \bar{\eta}_{s} \delta(l)^{T} H_{s}^{T} R_{1 s} H_{s} \delta(l) \\
& +\sum_{s=1}^{3} \sum_{\theta=-\bar{\eta}_{s}+1}^{-\eta_{s}} \sum_{l=k+\theta-1}^{k-1}\left(\bar{\eta}_{s}-\underline{\eta_{s}}\right) \delta(l)^{T} H_{s}^{T} R_{2 s} H_{s} \delta(l),
\end{aligned}
$$

$\delta(l)=x(l+1)-x(l), P(\mu(k), d(k), \tau(k)), Q_{1 s}, Q_{2 s}, Q_{3 s}, R_{1 s}$, $R_{2 s}>0, s=\{1,2,3\}, \bar{t}_{1}, \underline{t_{1}}, \bar{t}_{2}, t_{2}, \bar{t}_{3}$, and $\underline{t_{3}}$ are defined in Theorem 5. In the following, when $\mu(k)=\bar{i}, d(k)=m$, and $\tau(k)=p$, we will denote $P(\mu(k), d(k), \tau(k)), \bar{A}(\mu(k))$ and $\bar{B}_{s}(\mu(k))$ by $P_{i, m, p}, \bar{A}_{i}$ and $\bar{B}_{i s}$, respectively.

$$
\text { Let } \xi(k)=\left[x(k)^{T} \eta(k)^{T} \bar{\eta}(k)^{T} \underline{\eta}(k)^{T}\right]^{T} \text {, }
$$

$\eta(k)$

$=\left[\begin{array}{ll}\left(H_{1} x(k-m)\right)^{T} & \left(H_{2} x(k-p)\right)^{T}\left(H_{3} x(k-m-p)\right)^{T}\end{array}\right]^{T}$,

$\underline{\eta}(k)$

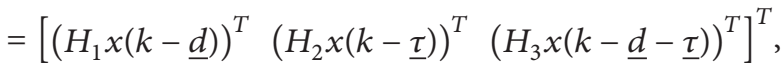

$\bar{\eta}(k)$

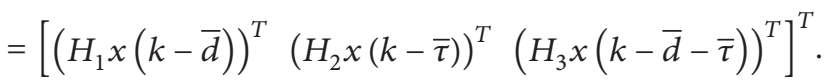

Then, along the solution of system (5) we have

$$
\begin{aligned}
\mathbb{E}\left[\Delta V_{1}(k)\right]= & x(k+1)^{T}\left[\sum_{j=1}^{s_{1}} \sum_{n=0}^{s_{2}} \sum_{q=0}^{s_{3}} \pi_{i j} \lambda_{m n} \gamma_{p q} P_{j, n, q}\right] \\
& \times x(k+1)-x^{T}(k) P_{i, m, p} x(k) \\
= & \xi^{T}(k)\left[\begin{array}{c}
\bar{A}_{i}^{T} \\
\Lambda^{T} \\
0 \\
0
\end{array}\right] \bar{P}_{i, m, p}\left[\begin{array}{llll}
\bar{A}_{i} & \Lambda & 0 & 0
\end{array}\right] \\
& \times \xi(k)-x^{T}(k) P_{i, m, p} x(k),
\end{aligned}
$$

where $\bar{P}_{i, m, p}$ and $\Lambda$ are defined in Theorem 5 .

Consider that

$$
\begin{aligned}
\mathbb{E}\left[\Delta V_{2}(k)\right]= & \sum_{s=1}^{3}\left(\left(H_{s} x(k)\right)^{T}\left(Q_{1 s}+Q_{2 s}\right) H_{s} x(k)\right. \\
& -\left(H_{s} x\left(k-\bar{t}_{s}\right)\right)^{T} Q_{1 s} H_{s} x\left(k-\bar{t}_{s}\right) \\
& \left.-\left(H_{s} x\left(k-\underline{t_{s}}\right)\right)^{T} Q_{2 s} H_{s} x\left(k-\underline{t_{s}}\right)\right),
\end{aligned}
$$




$$
\begin{aligned}
& \mathbb{E}\left[\Delta V_{3}(k)\right]=\sum_{s=1}^{3}\left(\left(\bar{t}_{s}-\underline{t_{s}}+1\right) x(k)^{T} H_{s}^{T} Q_{3 s} H_{s} x(k)\right. \\
&-\sum_{\theta=k-\bar{t}_{s}+1}^{k-t_{s}} x(l)^{T} H_{s}^{T} Q_{3 s} H_{s} x(l) \\
&+\left(\sum_{l=k-\eta_{s}(k+1)+1}^{k-1}-\sum_{l=k-\eta_{s}(k)+1}^{k-1}\right) \\
& \times x(l)^{T} H_{s}^{T} Q_{3 s} H_{s} x(l) \\
&-x\left(k-\eta_{s}(k)\right)^{T} \\
&\left.\times H_{s}^{T} Q_{3 s} H_{s} x\left(k-\eta_{s}(k)\right)\right) .
\end{aligned}
$$

Note that

$$
\begin{aligned}
& \sum_{s=1}^{3} \sum_{l=k-\eta_{s}(k+1)+1}^{k-1} x^{T}(l) H_{s}^{T} Q_{3 s} H_{s} x(l) \\
& \quad=\sum_{s=1}^{3}\left[\sum_{l=k-t_{s}+1}^{k-1}+\sum_{l=k-\eta_{s}(k+1)+1}^{k-t_{s}}\right] x^{T}(l) H_{s}^{T} Q_{3 s} H_{s} x(l) \\
& \quad \leq \sum_{s=1}^{3}\left[\sum_{l=k-\eta_{s}(k)+1}^{k-1}+\sum_{l=k-\bar{t}_{s}+1}^{k-t_{s}}\right] x^{T}(l) H_{s}^{T} Q_{3 s} H_{s} x(l) .
\end{aligned}
$$

By combining (A.6) and (A.7), we have

$$
\begin{gathered}
\mathbb{E}\left[\Delta V_{3}\right]=\sum_{s=1}^{3}\left(\left(\bar{t}_{s}-\underline{t}_{s}+1\right) x(k)^{T} H_{s}^{T} Q_{3 s} H_{s} x(k)\right. \\
\left.-x\left(k-\eta_{s}(k)\right)^{T} H_{s}^{T} Q_{3 s} H_{s} x\left(k-\eta_{s}(k)\right)\right), \\
\mathbb{E}\left[\Delta V_{4}\right]=\sum_{s=1}^{3}\left(\begin{array}{c}
\bar{t}_{s}^{2} \delta^{T}(k) H_{s}^{T} R_{1 s} H_{s} \delta(k) \\
\quad-\sum_{l=k-\bar{t}_{s}}^{k-1} \bar{t}_{s} \delta^{T}(l) H_{s}^{T} R_{1 s} H_{s} \delta(l) \\
+\left(\bar{t}_{s}-\underline{t}_{s}\right)^{2} \delta^{T}(k) H_{s}^{T} R_{2 s} H_{s} \delta(k) \\
\left.\quad-\sum_{l=k-\bar{t}_{s}}^{k-1}\left(\bar{t}_{s}-t_{s}\right) \delta^{T}(l) H_{s}^{T} R_{2 s} H_{s} \delta(l)\right)
\end{array}\right)
\end{gathered}
$$

By Jensen's inequality, we can get

$$
\begin{aligned}
& \sum_{s=1}^{3} \sum_{l=k-\bar{t}_{s}}^{k-1} \bar{t}_{s} \delta^{T}(l) H_{s}^{T} R_{1 s} H_{s} \delta(l) \\
& =\sum_{s=1}^{3}\left(\sum_{l=k-\bar{t}_{s}}^{k-\eta_{k}-1}+\sum_{l=k-\eta_{k}}^{k-1}\right) \\
& \times\left(\bar{t}_{s}-\eta_{k}+\eta_{k}\right) \delta^{T}(l) H_{s}^{T} R_{1 s} H_{s} \delta(l) \\
& \geq \sum_{s=1}^{3}\left(\left(x\left(k-\bar{t}_{s}\right)-x\left(k-\eta_{k}\right)\right)^{T} H_{s}^{T} R_{1 s} H_{s}\right. \\
& \times\left(x\left(k-\bar{t}_{s}\right)-x\left(k-\eta_{k}\right)\right) \\
& +\left(x\left(k-\eta_{k}\right)-x(k)\right)^{T} \\
& \left.\times H_{s}^{T} R_{1 s} H_{s}\left(x\left(k-\eta_{k}\right)-x(k)\right)\right) \\
& \geq \xi(k)^{T}\left[\begin{array}{cccc}
\Omega_{11} & -\Omega_{12} & 0 & 0 \\
* & \Omega_{22} & 0 & -\Omega_{24} \\
* & * & 0 & 0 \\
* & * & * & \Omega_{44}
\end{array}\right] \xi(k),
\end{aligned}
$$

where $\Omega_{11}, \Omega_{12}, \Omega_{22}, \Omega_{24}$, and $\Omega_{44}$ are defined in Theorem 5 . Similarly, we have

$$
\begin{aligned}
& \sum_{s=1}^{3} \sum_{l=k-\bar{t}_{s}}^{k-t_{s}-1}\left(\bar{t}_{s}-\underline{t}_{s}\right) \delta^{T}(l) H_{s}^{T} R_{2 s} H_{s} \delta(l) \\
& =\sum_{s=1}^{3}\left(\sum_{l=k-\bar{t}_{s}}^{k-\eta_{k}-1}+\sum_{l=k-\eta_{k}}^{k-t_{s}-1}\right) \\
& \times\left(\bar{t}_{s}-\eta_{k}+\eta_{k}-\underline{t_{s}}\right) \delta^{T}(l) H_{s}^{T} R_{2 s} H_{s} \delta(l) \\
& \geq \sum_{s=1}^{3}\left(\left(\bar{t}_{s}-\eta_{k}\right) \sum_{l=k-\bar{t}_{s}}^{k-\eta_{k}-1} \delta^{T}(l) H_{s}^{T} R_{2 s} H_{s} \delta(l)\right. \\
& \left.+\left(\eta_{k}-\underline{t_{s}}\right) \sum_{l=k-\eta_{k}}^{k-t_{s}-1} \delta^{T}(l) H_{s}^{T} R_{2 s} H_{s} \delta(l)\right) \\
& \geq \sum_{s=1}^{3}\left(\left(\sum_{l=k-\bar{t}_{s}}^{k-\eta_{k}-1} \delta(l)\right)^{T} H_{s}^{T} R_{2 s} H_{s}\left(\sum_{l=k-\bar{t}_{s}}^{k-\eta_{k}-1} \delta(l)\right)\right. \\
& \left.+\left(\sum_{l=k-\eta_{k}}^{k-t_{s}-1} \delta(l)\right)^{T} H_{s}^{T} R_{2 s} H_{s}\left(\sum_{l=k-\eta_{k}}^{k-t_{s}-1} \delta(l)\right)\right) \\
& \geq \xi(k)^{T}\left[\begin{array}{cccc}
0 & 0 & 0 & 0 \\
* & 2 \Psi & -\Psi & -\Psi \\
* & * & \Psi & 0 \\
* & * & * & \Psi
\end{array}\right] \xi(k)
\end{aligned}
$$

where $\Psi$ is defined in Theorem 5 . 
By combining (A.9), (A.10), and (A.11), we have

$\mathbb{E}\left[\Delta V_{4}\right]$

$$
\begin{aligned}
& \leq \xi(k)^{T}\left\{\left[\begin{array}{c}
\bar{t}_{1}\left(H_{1}\left(\bar{A}_{i}-I\right)\right)^{T} \\
\bar{t}_{1} \bar{\Lambda}^{T} \\
0 \\
0
\end{array}\right]\right. \\
& \times R_{11}\left[\bar{t}_{1} H_{1}\left(\bar{A}_{i}-I\right) \bar{t}_{1} \bar{\Lambda} \quad 0 \quad 0\right] \\
& +\left[\begin{array}{c}
\bar{t}_{2}\left(H_{2}\left(\bar{A}_{i}-I\right)\right)^{T} \\
\bar{t}_{2} \bar{\Lambda}^{T} \\
0 \\
0
\end{array}\right] \\
& \times R_{12}\left[\bar{t}_{2} H_{2}\left(\bar{A}_{i}-I\right) \bar{t}_{1} \bar{\Lambda} \quad 0 \quad 0\right] \\
& +\left[\begin{array}{c}
\bar{t}_{3}\left(H_{3}\left(\bar{A}_{i}-I\right)\right)^{T} \\
\bar{t}_{3} \bar{\Lambda}^{T} \\
0 \\
0
\end{array}\right] \\
& \times R_{13}\left[\bar{t}_{3} H_{3}\left(\bar{A}_{i}-I\right) \bar{t}_{1} \bar{\Lambda} \quad 0 \quad 0\right] \\
& +\left[\begin{array}{c}
\left(\bar{t}_{1}-\frac{t_{1}}{}\right)\left(H_{1}\left(\bar{A}_{i}-I\right)\right)^{T} \\
\left(\bar{t}_{1}-t_{1}\right) \\
0 \\
0
\end{array}\right] \\
& \times R_{21}\left[\left(\bar{t}_{1}-\underline{t_{1}}\right) H_{1}\left(\bar{A}_{i}-I\right)\left(\bar{t}_{1}-\underline{t_{1}}\right) \bar{\Lambda} \quad 000\right] \\
& +\left[\begin{array}{c}
\left(\bar{t}_{2}-\frac{t_{2}}{\underline{2}}\right)\left(H_{2}\left(\bar{A}_{i}-I\right)\right)^{T} \\
\left(\bar{t}_{2}-\frac{t_{2}}{0}\right) \bar{\Lambda}^{T} \\
0
\end{array}\right] \\
& \times R_{22}\left[\left(\bar{t}_{2}-\underline{t_{2}}\right) H_{2}\left(\bar{A}_{i}-I\right)\left(\bar{t}_{2}-\underline{t_{2}}\right) \bar{\Lambda} \quad 000\right] \\
& +\left[\begin{array}{c}
\left(\bar{t}_{3}-\frac{t_{3}}{\bar{t}_{3}}\right)\left(H_{3}\left(\bar{A}_{i}-I\right)\right)^{T} \\
\left(\bar{t}_{3}-t_{3}\right) \bar{\Lambda}^{T} \\
0
\end{array}\right] \\
& \times R_{23}\left[\left(\bar{t}_{3}-\underline{t_{3}}\right) H_{3}\left(\bar{A}_{i}-I\right)\left(\bar{t}_{3}-\underline{t_{3}}\right) \bar{\Lambda} \quad 000\right] \\
& +\left[\begin{array}{cccc}
-\Omega_{11} & \Omega_{12} & 0 & 0 \\
* & -\Omega_{22} & 0 & \Omega_{24} \\
* & * & 0 & 0 \\
* & * & * & -\Omega_{44}
\end{array}\right]
\end{aligned}
$$

$$
\left.+\left[\begin{array}{cccc}
0 & 0 & 0 & 0 \\
* & -2 \Psi & \Psi & \Psi \\
* & * & -\Psi & 0 \\
* & * & * & -\Psi
\end{array}\right]\right\} \xi(k)
$$

where $\bar{\Lambda}$ is defined in Theorem 5 .

By combining (A.4), (A.5), (A.8), and (A.12), we have $\mathbb{E}[\Delta V]$

$$
\leq \xi^{T}(k)
$$

$$
\times\left\{\left[\begin{array}{cccc}
\Pi_{i, m, p} & \Omega_{12} & 0 & 0 \\
* & -\bar{Q}_{3}-\Omega_{22}-2 \Psi & \Psi & \Omega_{24}+\Psi \\
* & * & -\bar{Q}_{2}-\Psi & 0 \\
* & * & * & -\bar{Q}_{1}-\Omega_{44}-\Psi
\end{array}\right]\right.
$$$$
+\left[\begin{array}{c}
\bar{A}_{i}^{T} \\
\Lambda^{T} \\
0 \\
0
\end{array}\right] \bar{P}_{i, m, p}\left[\begin{array}{llll}
\bar{A}_{i} & \Lambda & 0 & 0
\end{array}\right]
$$$$
+\left[\begin{array}{c}
\bar{t}_{1}\left(H_{1}\left(\bar{A}_{i}-I\right)\right)^{T} \\
\bar{t}_{1} \bar{\Lambda}^{T} \\
0 \\
0
\end{array}\right]
$$$$
\times R_{11}\left[\bar{t}_{1} H_{1}\left(\bar{A}_{i}-I\right) \bar{t}_{1} \bar{\Lambda} \quad 0 \quad 0\right]
$$$$
+\left[\begin{array}{c}
\bar{t}_{2}\left(H_{2}\left(\bar{A}_{i}-I\right)\right)^{T} \\
\bar{t}_{2} \bar{\Lambda}^{T} \\
0 \\
0
\end{array}\right]
$$$$
\times R_{12}\left[\bar{t}_{2} H_{2}\left(\bar{A}_{i}-I\right) \bar{t}_{2} \bar{\Lambda} 00\right]
$$$$
+\left[\begin{array}{c}
\bar{t}_{3}\left(H_{3}\left(\bar{A}_{i}-I\right)\right)^{T} \\
\bar{t}_{3} \bar{\Lambda}^{T} \\
0 \\
0
\end{array}\right]
$$$$
\times R_{13}\left[\bar{t}_{3} H_{3}\left(\bar{A}_{i}-I\right) \bar{t}_{3} \bar{\Lambda} \quad 0 \quad 0\right]
$$$$
+\left[\begin{array}{c}
\left(\bar{t}_{1}-\frac{\left.t_{1}\right)}{\left.\bar{t}_{\bar{t}_{1}}-t_{1}\right)}\left(H_{1}\left(\bar{A}_{i}-I\right)\right)^{T}\right. \\
0 \\
0
\end{array}\right]
$$$$
\times R_{21}\left[\left(\bar{t}_{1}-t_{1}\right) H_{1}\left(\bar{A}_{i}-I\right)\left(\bar{t}_{1}-\underline{t_{1}}\right) \bar{\Lambda} \quad 000\right]
$$$$
+\left[\begin{array}{c}
\left(\bar{t}_{2}-\frac{\left.t_{2}\right)}{\left(\bar{t}_{2}-\frac{t_{2}}{2}\right)}\left(\bar{A}_{i}-I\right)\right)^{T} \\
0 \\
0
\end{array}\right]
$$ 


$$
\begin{aligned}
& \times R_{22}\left[\left(\bar{t}_{2}-\underline{t_{2}}\right) H_{2}\left(\bar{A}_{i}-I\right)\left(\bar{t}_{2}-\underline{t_{2}}\right) \bar{\Lambda} \quad 000\right] \\
& +\left[\begin{array}{c}
\left(\bar{t}_{3}-\frac{t_{3}}{\underline{3}}\right)\left(H_{3}\left(\bar{A}_{i}-I\right)\right)^{T} \\
\left(\bar{t}_{3}-\underline{t_{3}}\right) \bar{\Lambda}^{T} \\
0
\end{array}\right] \\
& \left.\times R_{23}\left[\left(\bar{t}_{3}-\underline{t_{3}}\right) H_{3}\left(\bar{A}_{i}-I\right)\left(\bar{t}_{3}-\underline{t_{3}}\right) \bar{\Lambda} \quad 000\right]\right\} \\
& \times \xi(k) \\
& =\xi^{T}(k) \Theta_{i, m, p} \xi(k),
\end{aligned}
$$

where $\bar{Q}_{1}, \bar{Q}_{2}, \bar{Q}_{3}$, and $\Pi_{i, m, p}$ are defined in Theorem 5 .

By Schur complement and from (10), we have $\Theta_{i, m, p}<0$. Therefore,

$$
\mathbb{E}[\Delta V] \leq-\lambda_{\min }\left(-\Theta_{i, m, p}\right) \xi(k)^{T} \xi(k) \leq-\eta x(k)^{T} x(k),
$$

where $\lambda_{\min }\left(-\Theta_{i, m, p}\right)$ denotes the minimal eigenvalue of $-\Theta_{i, m, p}$ and $\eta=\inf \left\{\lambda_{\text {min }}\left(-\Theta_{i, m, p}\right)\right\}$. From (A.14), it is seen that for any $t>0$

$$
\mathbb{E}[V(k+1)]-\mathbb{E}[V(0)] \leq-\eta \sum_{k=0}^{t} E\left[x(k)^{T} x(k)\right]
$$

Furthermore

$$
\sum_{k=0}^{t} \mathbb{E}\left[x(k)^{T} x(k)\right] \leq \frac{1}{\eta} \mathbb{E}[V(0)]
$$

By taking limit as $t \rightarrow \infty$, we have

$$
\sum_{k=0}^{\infty} \mathbb{E}\left[x(k)^{T} x(k)\right] \leq \frac{1}{\eta} \mathbb{E}[V(0)]<\infty .
$$

According to Definition 4, the closed-loop system (5) is stochastically stable. This completes the proof.

\section{Acknowledgments}

This work is supported by the National Natural Science Foundation of China (Grant nos. 61174070, 61104107), the Foundation of Key Laboratory of Autonomous Systems and Networked Control, Ministry of Education, P.R. China, the Natural Science Foundation of Guangdong Province (Grant no. S2011040004702), China Postdoctoral Science Foundation (Grant no. 20110491334), the Project-Sponsored by SRF for ROCS, SEM (Grant no. 20100172120028), and the Foundation of Shenzhen University (Grant nos. JG2012035,00035653).

\section{References}

[1] W. Zhang, M. S. Branicky, and S. M. Phillips, "Stability of networked control systems," IEEE Control Systems Magazine, vol. 21, no. 1, pp. 84-99, 2001.

[2] Y. Tipsuwan and M. Y. Chow, "Control methodologies in networked control systems," Control Engineering Practice, vol. 11, no. 10, pp. 1099-1111, 2003.

[3] T. C. Yang, "Networked control system: a brief survey," IEE Proceedings, vol. 153, no. 4, pp. 403-412, 2006.

[4] J. P. Hespanha, P. Naghshtabrizi, and Y. Xu, "A survey of recent results in networked control systems," Proceedings of the IEEE, vol. 95, no. 1, pp. 138-172, 2007.

[5] M. C. F. Donkers, W. P. M. H. Heemels, N. van de Wouw, and L. Hetel, "Stability analysis of networked control systems using a switched linear systems approach," IEEE Transactions on Automatic Control, vol. 56, no. 9, pp. 2101-2115, 2011.

[6] L. X. Zhang, H. J. Gao, and O. Kaynak, "Network-induced constraints in networked control systems-a survey," IEEE Transactions on Automatic Control, vol. 9, no. 1, pp. 403-416, 2013.

[7] F. Rasool, D. Huang, and S. K. Nguang, "Robust $H_{\infty}$ output feedback control of networked control systems with multiple quantizers," Journal of the Franklin Institute, vol. 349, no. 3, pp. 1153-1173, 2012.

[8] W. P. M. H. Heemels, A. R. Teel, N. van de Wouw, and D. Nešić, "Networked control systems with communication constraints: tradeoffs between transmission intervals, delays and performance," IEEE Transactions on Automatic Control, vol. 55, no. 8, pp. 1781-1796, 2010.

[9] J. Xiong and J. Lam, "Stabilization of linear systems over networks with bounded packet loss," Automatica, vol. 43, no. 1, pp. 80-87, 2007.

[10] Z. Wang, F. Yang, D. W. C. Ho, and X. Liu, "Robust $H_{\infty}$ control for networked systems with random packet losses," IEEE Transactions on Systems, Man, and Cybernetics Part B, vol. 37, no. 4, pp. 916-924, 2007.

[11] J. Wu and T. Chen, "Design of networked control systems with packet dropouts," IEEE Transactions on Automatic Control, vol. 52, no. 7, pp. 1314-1319, 2007.

[12] C.-C. Hsieh and P.-L. Hsu, "Analysis and applications of the motion message estimator for network control systems," Asian Journal of Control, vol. 10, no. 1, pp. 45-54, 2008.

[13] T. Jia, Y. Niu, and X. Wang, " $H_{\infty}$ control for networked systems with data packet dropout," International Journal of Control, Automation and Systems, vol. 8, no. 2, pp. 198-203, 2010.

[14] Q. G. Lu, L. X. Zhang, M. Basin, and H. Tian, "Analysis and synthesis for networked control systems with uncertain rate of packet losses," Journal of the Franklin Institute, vol. 349, no. 7, pp. 2500-2514, 2012.

[15] W.-A. Zhang, L. Yu, and S. Yin, "A switched system approach to $H_{\infty}$ control of networked control systems with time-varying delays," Journal of the Franklin Institute, vol. 348, no. 2, pp. 165178, 2011.

[16] E. G. Tian and D. Yue, "A new state feedback $H_{\infty}$ control of networked control systems with time-varying network conditions," Journal of the Franklin Institute, vol. 349, no. 3, pp. 891-914, 2012.

[17] L. Qiu, B. G. Xu, and S. B. Li, " $H_{2} / H_{\infty}$ control of networked control system with random time delays," Science China, vol. 54, no. 12 , pp. 2615-2630, 2011. 
[18] D. Yue, E. Tian, Z. Wang, and J. Lam, "Stabilization of systems with probabilistic interval input delays and its applications to networked control systems," IEEE Transactions on Systems, Man, and Cybernetics Part A, vol. 39, no. 4, pp. 939-945, 2009.

[19] Y. Shi and B. Yu, "Output feedback stabilization of networked control systems with random delays modeled by Markov chains," IEEE Transactions on Automatic Control, vol. 54, no. 7, pp. 1668-1674, 2009.

[20] M. B. G. Cloosterman, N. van de Wouw, W. P. M. H. Heemels, and H. Nijmeijer, "Stability of networked control systems with uncertain time-varying delays," IEEE Transactions on Automatic Control, vol. 54, no. 7, pp. 1575-1580, 2009.

[21] S. Hu and Q. Zhu, "Stochastic optimal control and analysis of stability of networked control systems with long delay," Automatica, vol. 39, no. 11, pp. 1877-1884, 2003.

[22] F. Yang, Z. Wang, Y. S. Hung, and M. Gani, " $H_{\infty}$ control for networked systems with random communication delays," IEEE Transactions on Automatic Control, vol. 51, no. 3, pp. 511-518, 2006.

[23] C. Peng and D. Yue, "State feedback controller design of networked control systems with parameter uncertainty and state-delay," Asian Journal of Control, vol. 8, no. 4, pp. 385-392, 2006.

[24] D. Yue, E. G. Tian, and Q. L. Han, "A delay system method for designing event-triggered controllers of networked control systems," IEEE Transactions on Automatic Control, vol. 58, no. 2, pp. 475-481, 2013.

[25] R. W. Brockett and D. Liberzon, "Quantized feedback stabilization of linear systems," IEEE Transactions on Automatic Control, vol. 45, no. 7, pp. 1279-1289, 2000.

[26] D. F. Delchamps, "Stabilizing a linear system with quantized state feedback," IEEE Transactions on Automatic Control, vol. 35, no. 8, pp. 916-924, 1990.

[27] D. Liberzon, "On stabilization of linear systems with limited information," IEEE Transactions on Automatic Control, vol. 48, no. 2, pp. 304-307, 2003.

[28] Y. Ishido, K. Takaba, and D. E. Quevedo, "Stability analysis of networked control systems subject to packet-dropouts and finite-level quantization," Systems \& Control Letters, vol. 60, no. 5, pp. 325-332, 2011.

[29] Y. Tang, H. J. Gao, W. Zou, and J. Kurths, "Distributed synchronization in networks of agent systems with nonlinearities and random switchings," IEEE Transactions on Systems, Man, and Cybernetics Part B, vol. 43, pp. 358-370, 2013.

[30] E. Tian, D. Yue, and C. Peng, "Reliable control for networked control systems with probabilistic sensors and actuators faults," IET Control Theory and Applications, vol. 4, no. 8, pp. 1478-1488, 2010.

[31] L. A. Montestruque and P. Antsaklis, "Stability of model-based networked control systems with time-varying transmission times," IEEE Transactions on Automatic Control, vol. 49, no. 9, pp. 1562-1572, 2004.

[32] M. Izá, D. Görges, and S. Liu, "Optimal control of networked control systems with uncertain time-varying transmission delay," in Proceedings of the 2nd IFAC Workshop on Distributed Estimation and Control in Networked Systems, pp. 13-18, Annecy, France, 2010.

[33] H. Fujioka, "A discrete-time approach to stability analysis of systems with aperiodic sample-and-hold devices," IEEE Transactions on Automatic Control, vol. 54, no. 10, pp. 2440-2445, 2009.
[34] T. Suzuki, M. Kono, N. Takahashi, and O. Sato, "Controllability and stabilizability of a networked control system with periodic communication constraints," Systems \& Control Letters, vol. 60, no. 12, pp. 977-984, 2011.

[35] H. Rehbinder and M. Sanfridson, "Scheduling of a limited communication channel for optimal control," Automatica, vol. 40, no. 3, pp. 491-500, 2004.

[36] X. F. Wu, W. H. Gui, and J. J. He, "Stability analysis of networked control system with control channels constraints and time delay," International Journal of Digital Content Technology and Its Applications, vol. 6, no. 7, pp. 151-158, 2012.

[37] Z. H. Guan, C. Y. Chen, G. Feng, and T. Li, "Optimal tracking performance limitation of networked control systems with limited bandwidth and additive colored white Gaussian noise," IEEE Transactions on Circuits and Systems-I, vol. 60, no. 1, pp. 189-198, 2013.

[38] W.-A. Zhang and L. Yu, "Modelling and control of networked control systems with both network-induced delay and packetdropout," Automatica, vol. 44, no. 12, pp. 3206-3210, 2008.

[39] T. Y. Yu, L. Wang, and M. Yu, "Switched system approach to stabilization of networked control systems," International Journal of Robust and Nonlinear Contro, vol. 21, no. 17, pp. 19151946, 2011.

[40] Y.-L. Wang and G.-H. Yang, "State feedback control synthesis for networked control systems with packet dropout," Asian Journal of Control, vol. 11, no. 1, pp. 49-58, 2009.

[41] B. Yu and Y. Shi, "State feedback stabilization of networked control systems with random time delays and packet dropout," in Proceedings of the ASME Dynamic Systems and Control Conference (DSCC '08), pp. 127-133, October 2008.

[42] H. Dong, Z. Wang, and H. Gao, "Robust $H_{\infty}$ filtering for a class of nonlinear networked systems with multiple stochastic communication delays and packet dropouts," IEEE Transactions on Signal Processing, vol. 58, no. 4, pp. 1957-1966, 2010.

[43] Y.-L. Wang and G.-H. Yang, "Time delay and packet dropout compensation for networked control systems: a linear estimation method," International Journal of Control, vol. 83, no. 1, pp. 115-124, 2010.

[44] A. Liu, L. Yu, and W. A. Zhang, "One-step receding horizon $H_{\infty}$ control for networked control systems with random delay and packet disordering," ISA Transactions, vol. 50, no. 1, pp. 44-52, 2011.

[45] O. L. V. Costa, M. D. Fragoso, and R. P. Marques, Discrete-Time Markov Jump Linear Systems, Springer, 2005.

[46] L. Zhang, Y. Shi, T. Chen, and B. Huang, "A new method for stabilization of networked control systems with random delays," IEEE Transactions on Automatic Control, vol. 50, no. 8, pp. 11771181, 2005.

[47] J. Nilsson, Real-time Control Systems with Delays, Lund Institute of Technology, Lund, Sweden, 1998.

[48] L. Xiao, A. Hassibi, and J. P. How, "Control with random communication delays via a discrete-time jump system approach," in Proceedings of the American Control Conference, pp. 21992204, June 2000.

[49] J. Wu, T. Chen, and $\mathrm{S} . \mathrm{Xu}$, "Stochastic stabilization and $H_{\infty}$ control for discrete jumping systems with time delays," Asian Journal of Control, vol. 7, no. 3, pp. 223-230, 2005. 


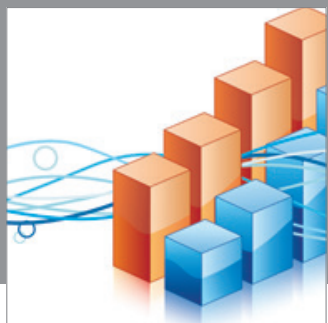

Advances in

Operations Research

mansans

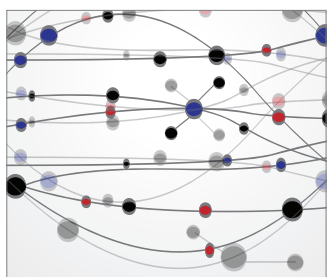

The Scientific World Journal
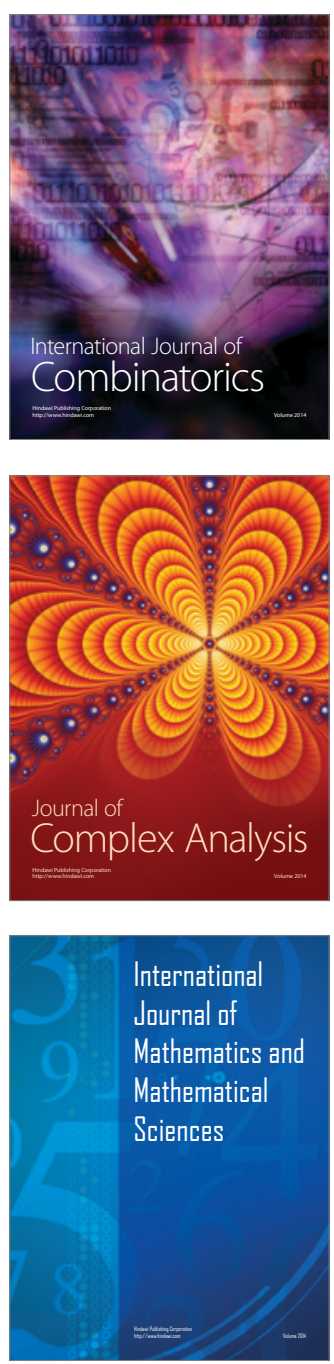
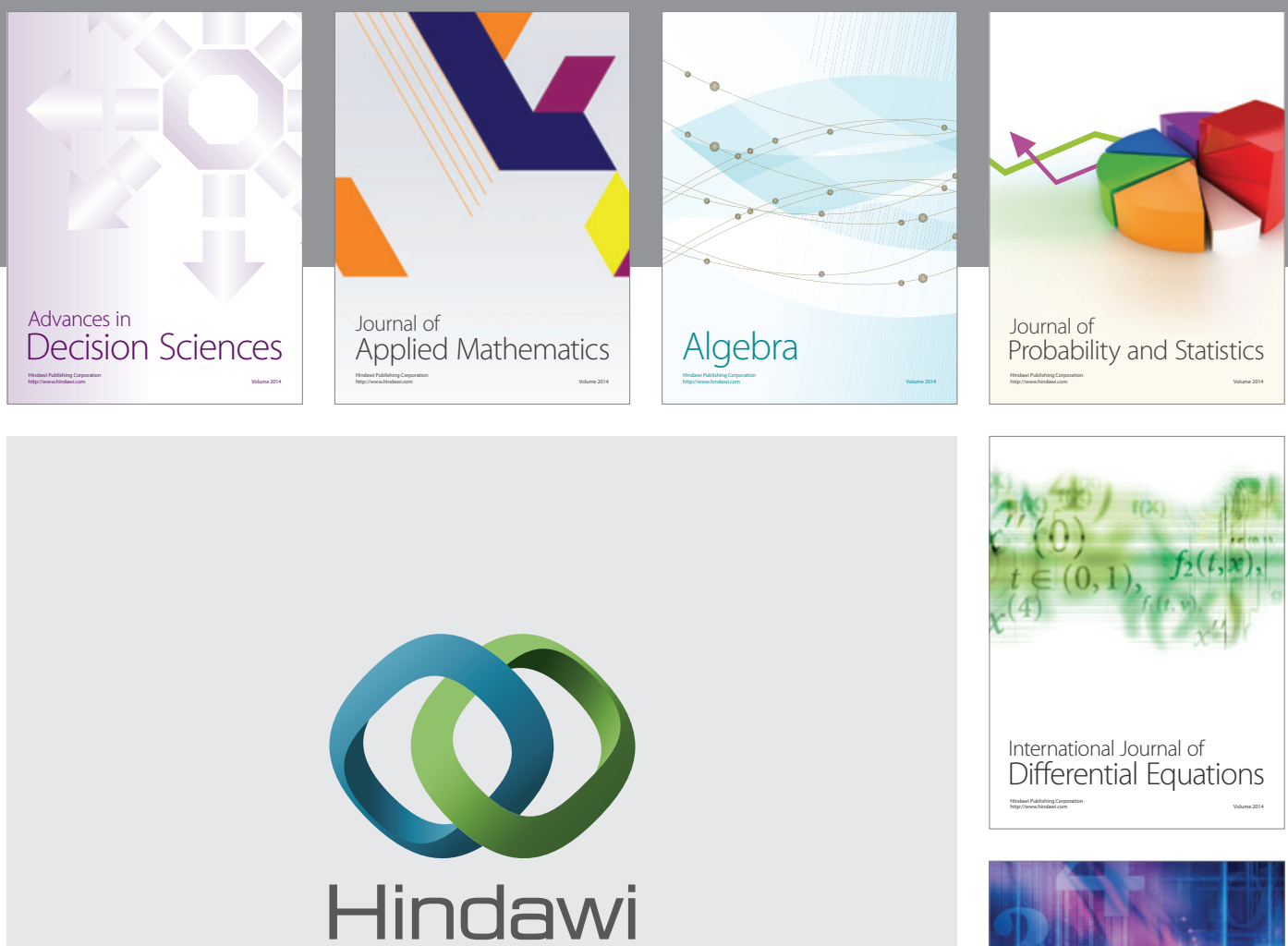

Submit your manuscripts at http://www.hindawi.com
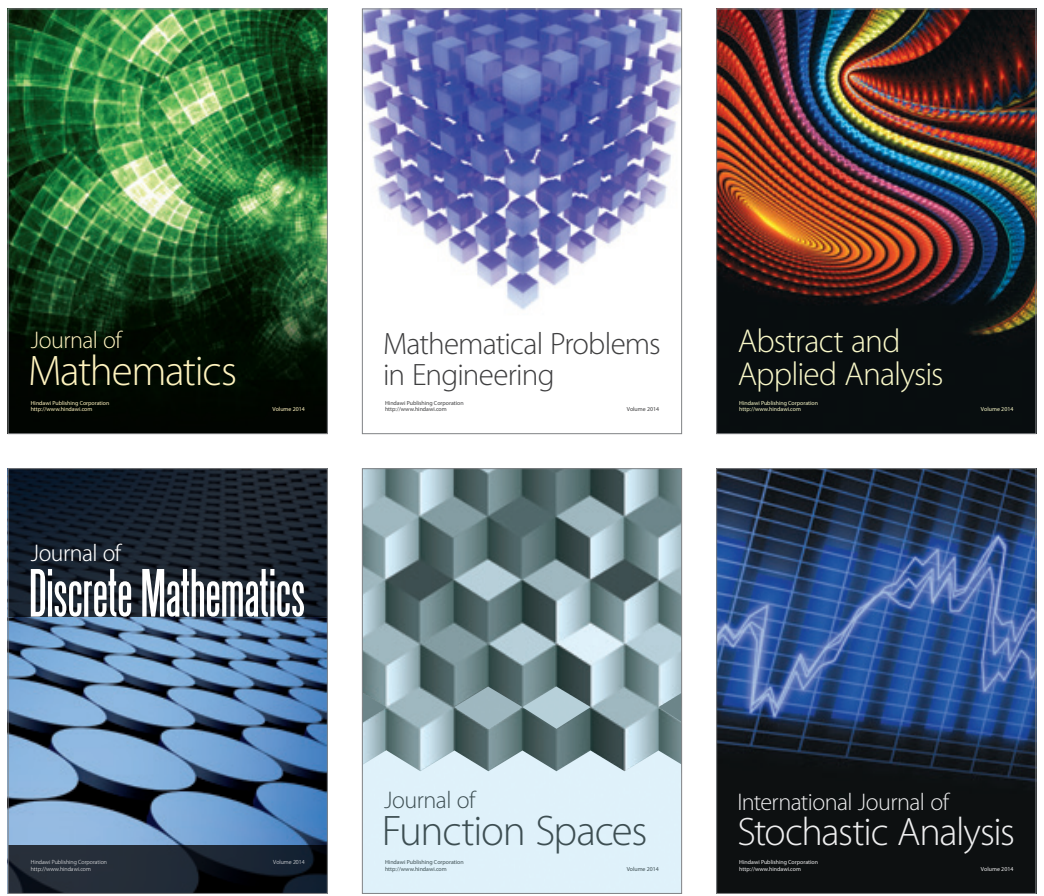

Journal of

Function Spaces

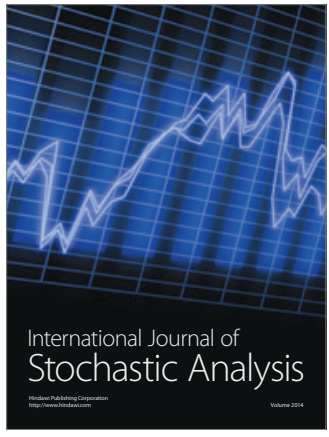

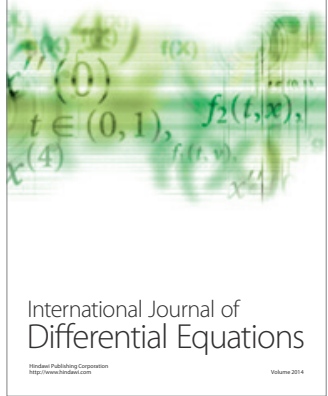
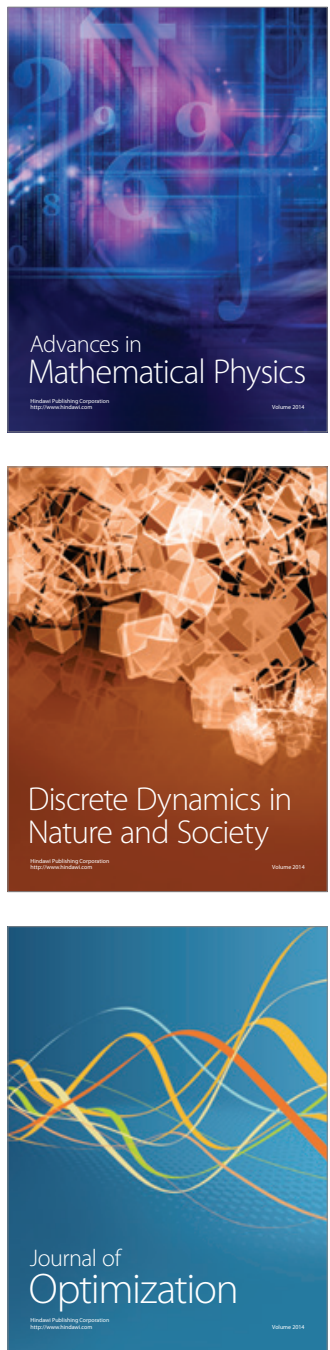